\title{
A Cessibilidade em ambiente Universitário: IDENTIFICAÇão de BARREIRAS ARQUITETÔNICAS NO CAMPUS DA USP DE BAURU ${ }^{1}$
}

\author{
A CCESSIBILITY IN THE UNIVERSITY ENVIRONMENT: IDENTIFICATION OF \\ ARCHITECTURAL BARRIERS IN THE USP CAMPUS OF BAURU
}

\author{
Dionísia A parecida Cusin LAMÔNICA ${ }^{2}$ \\ Pedro ARAÚJO-FILHO ${ }^{3}$ \\ Simone Berriel Joaquim SIMOMELLI ${ }^{4}$ \\ Vera Lígia Santos Butiquiol CAETANO ${ }^{5}$ \\ Márcia Regina Rodrigues REGIN A ${ }^{6}$ \\ Denise Maria REGIANI ${ }^{7}$
}

RESU M O: a acessibilidade é um direito do cidadão assegurado por lei para que portadores de deficiência
tenham a possi bilidade de usufruir de recursos e ações no âmbito social. Barreiras arquitetônicas interferem na
vida destes podendo deixá-los a parte da convivência evida social. Os objetivos deste estudo foram: identificar,
descrever e mapear barreiras físicas no Campus da Universidade de São Paulo de Bauru e apresentar as
intervenções realizadas, durante o período de outubro de 2001 a dezembro de 2005 . O estudo foi descritivo-
quantitativo, no qual a coleta de dados centrou-se na análise das condições arquitetônicas das três unidades
que compõe este campus, observada as normativas da Associação Brasileira de Normas técnicas e realizada
intervenções. Foram identificados: 72 pontos de guias não rebaixadas, 21 pontos de acessos com diferenças de
níveis sem rampas; $220 m^{2}$ de escadas/ rampas sem corrimãos; $658 m^{2}$ de escadas/ rampas com corrimãos em
discordância com as normas vigentes; 03 rampas com inclinações superiores às determinadas nas normas
técnicas; 10 banheiros parcial mente adaptados para deficientes; 02 vagas de estacionamento parcial mente
adaptadas e 02 elevadores existentes. Foram executados, em 19 pontos, rebaixamentos de guias, somando 115

${ }^{1}$ Todos os autores foram Membros da Comissão para Assuntos relativos a pessoas portadoras de Deficiência da PCAB durante o período de outubro de 2001 a dezembro de 2005

2 Professora Livre Docente, Associada do Departamento de Fonoaudiologia da FOB/ USP, Presidente da Comissão Interna para A ssuntos Relativos às Pessoas Portadoras de Deficiência no Campus da Universidade São Paulo de Bauru. Via Puccini 1-16; Residencial Tívoli I, Bairro Tívoli I, Bauru/ SP CEP: 17043095; e-mail: dionelam@uol.com.br

${ }^{3}$ Desenhista da Divisão de Infra-estrutura da PCAB, representante do Programa USP Legal - Campus de Bauru. M embro da Comissão Interna para A ssuntos Relativos às Pessoas Portadoras de Deficiência no Campus da Universidade São Paulo de Bauru. Rua Constancio Moraes Souza, 2-40, Bauru - SP, CEP: 17027-643. e-mail: pedrofilo@pcab.usp.br

${ }^{4}$ Engenheira, Diretora da Divisão de Infra-estrutura da PCAB. Membro da Comissão Interna para Assuntos Relativos às Pessoas Portadoras de Deficiência no Campus da Universidade São Paulo de Bauru. Rua 13 de maio 23-54. Bauru/ SP CEP: 17016170. simone@pcab.usp.br

${ }^{5}$ Técnica de Recursos Humanos, Chefe do Serviço de Pessoal da FOB. Membro da Comissão Interna para Assuntos Relativos às Pessoas Portadoras de Deficiência no Campus da Universidade São Paulo de Bauru. Rua Moisés leme da Silva 5-40; Bairro Aeroporto; Bauru/ SP. CEP: 1707335. veral sbc@fob.usp.br

${ }^{6}$ Enfermeira, Diretora Técnica de Serviços Administrativos do HRAC/ USP. Membro da Comissão Interna para A ssuntos Relativos às Pessoas Portadoras de Deficiência no Campus da Universidade São Paulo de Bauru. A viador A ntonio Gomes M eirelles, 8-51 jardim A mérica, Bauru/ SP. CEP: 17017323. maregina@centrinho.usp.br

7 Técnica para assuntos administrativos da FOB/ USP. Membro da Comissão Interna para Assuntos Relativos às Pessoas Portadoras de Deficiência no Campus da Universidade São Paulo de Bauru. Rua Manoel Cruz 17-49 Bauru/ SP. CEP: 17013-032. de.maria@fob.usp.br 
$\mathrm{m}^{2}$, com linhas de piso tátil, pintados; 8 rampas; 14 pontos deescadas erampas externas instaladas com corrimãos e guarda-corpos, 5 vagas exclusivas no estacionamento; instalado 2 centrais de atendimento telefônico para surdo e adquiridas 3 cadeira de rodas. As intervenções real izadas contribuíram para mel horar a acessibilidade de portadores de deficiência no campus favorecendo a utilização dos recursos existentes neste espaço público.

PALAVRAS-CHAVE: acessibilidade; barreiras arquitetônicas; ensino superior; educação especial.

\begin{abstract}
A BSTRACT: all citizens have the right assured by law to accessible to enable people with disabilities to enjoy resources and activities in the social environment. Physical barriers intervene in their life and can exclude them from living with others. This study's aims were to identify, describe and map the physical barriers at the Campus of Bauru of the University of São Paulo and to introduce interventions undertaken from October 2001 to December 2005. This was a quantitative descriptive study, in which data was collected on the architectural conditions analysis of the three units that make up this campus. The norms established by the Associação Brasileira de Normas Técnicas (ABNT) were used as bases for accomplishing the interventions. Results: 72 locations were identified as having curb cut problems, 21 points of access were found to have interruptions and grade changes that required ramps; $220 \mathrm{~m}^{2}$ of stairs/ ramps had no handrails; $658 \mathrm{~m}^{2}$ of stairs/ ramps had handrails that were not in accord with requirements; 3 ramps had inclinations higher than stipulated by requirements of the ABNT; 10 bathrooms were partially adapted for people with disabilities; 2 parking spaces were partially adapted and there were only 2 elevators. Curb cuts were installed at 19 points, adding $115 \mathrm{~m}^{2}$ with tactile lines painted on the pavement; 8 ramps were installed; 14 handrails and guardrails were installed along external stairs and ramps, 5 reserved parking places were designated and marked; 2 central phones for deaf people were installed and 3 wheelchairs were acquired. The various interventions contributed to improve accessibility for people with disabilities on campus facilitating the use of existing resources of public space.
\end{abstract}

KEYWORD S: accessibility; physical barriers; higher education; special education.

\title{
1 INTRODUÇÃO
}

A acessi bilidade é definida como possibilidade e condições de alcance para utilização, com segurança e autonomia, dos espaços, mobiliário e equipamentos urbanos, das edificações, dos transportes edos sistemas emeios de comunicação por pessoas portadoras de deficiência ou com mobilidade reduzida (BRASIL, 1994; BRASIL, 1998).

Pela legislação brasileira, toda pessoa, incluindo aquelas que apresentam deficiências, têm direito ao acesso à educação, à saúde, ao lazer e ao trabal ho. Desta forma, as pessoas devem ser percebidas com igualdade, implicando assim no reconhecimento e atendimento de suas necessidades especificas (ANDRADE et al., 2007).

A cessi bilidade compõe o conceito decidadania, no qual os indivíduos têm direitos assegurados por lei que devem ser respeitados, entretanto, muitos destes direitos esbarram em barreiras arquitetônicas e sociais (MANZINI et al., 2003). Um espaço construído, quando acessível a todos, é capaz de oferecer oportunidades igualitárias a todos os usuários (BITTEN COURT et al., 2004).

Para avaliação das condições de acessi bilidade deve ser considerado o contexto, a missão, o compromisso com a comunidadee os aspectos políticos, sociais e financeiros (PAGLIUCA et al., 2007). 
Nesta perspectiva, reflexões sobre as dificuldades ao acesso pelas barreiras físicas são salutares, pois contribuem para o repensar de práticas e proposição de ações, que podem favorecer a promoção de saúde e qual idade de vida destes indivíduos, favorecendo a convivência e transformando atitudes e comportamentos, interferindo nas relações interpessoais e nos comportamentos das pessoas.

Barreiras arquitetônicas têm sido definidas como obstáculos construídos no meio urbano ou nos edifícios, queimpedem ou dificultam a livrecirculação das pessoas que sofrem de al guma incapacidade transitória ou permanente (EMMEL; CASTRO, 2003). Estas se caracterizam por obstáculos aos acessos internos ou externos existentes em edificações de uso público ou privado.

De acordo com o Manual de Acessibilidade da ABNT (2004), estes obstáculos são descritos como:

- Escadas sem corrimão e sem contraste de cor nos degraus;

- Ausência de corrimãos e/ ou guarda-corpos normatizados;

- Ausência de banheiros adaptados,

- Ausência de rampas de acesso para cadeirante;

- Pouca iluminação;

- Ausência de orelhão, extintores de incêndio e caixas de correio adaptados a altura compatível com usuários de cadeira de rodas (a 1m do chão), ausência de sinalização tátil no chão, identificação desse mobiliário urbano pelos deficientes visuais;

- Falta de manutenção de ruas e calçadas, bueiros sem tampa ou grades de proteção;

- Salas deaula, teatros, anfiteatros eginásios sem vagas ou espaços nos corredores entre as poltronas, carteiras, arquibancadas para cadeiras de rodas;

- Desníveis nas portas que sejam maiores que $5 \mathrm{~cm}$;

- Portas e corredores estreitos (menor que $85 \mathrm{~cm}$ ), catracas sem porta alternativa;

- Portas emperradas e com maçanetas roliças ao invés do tipo alavanca, principal mente em banheiros adaptados;

- Banheiros sem identificação escrita, ao invés desímbolo que designem o gênero (para identificação dos analfabetos) e em relevo (para deficientes visuais);

- Falta de abrigos para sol e chuva nos pontos de ônibus.

Em 1‥ de outubro de 2001, por meio da Portaria GR 3.304, foi criado na Universidade de São Paulo a Comissão Permanente para Assuntos Relativos às Pessoas Portadoras de Deficiências. Esta comissão instituiu o Programa USP Legal 
objetivando a criação e implantação de política, voltada a estas pessoas em consonância com as normativas e iniciativas nacionais e internacionais.

O Campus da Universidade de São Paulo em Bauru é formado pela Faculdade de Odontologia (FOB), o Hospital de Reabilitação de Anomalias Craniofaciais (HRAC) ea Prefeitura do Campus A dministrativo de Bauru (PCAB).

Em 21 de agosto de 2003, para priorizar ações de intervenção do Programa USP Legal no Campus da USP deBauru, foi instituída a Comissão Interna para Assuntos Relativos às Pessoas Portadoras de Deficiência no Campus da Universidade São Paulo de Bauru - CIARP, por meio da Portaria PCAB n. 016/ 2003, cujos membros são representativos das unidades envolvidas no campus de Bauru.

As atribuições desta comissão são: diagnosticar as barreiras arquitetônicas do Campus da USP de Bauru e seus entornos; propor intervenções para adequação das áreas externas e internas do Campus; analisar projetos de reforma e obras novas do Campus, objetivando o atendimento às normas vigentes; promover a divulgação do Programa, contribuindo para a conscientização da comunidade interna sobre os assuntos relativos à questão; contribuir para o desenvolvimento das atividades do Programa USP Legal.

Os trabal hos realizados pela CIA RP em parceria com o USP Legal, nessa primeira gestão, foram:

- Realização de diagnóstico da situação atual e estudo de necessidades para acessibilidade às edificações do Campus da USP de Bauru;

- Aquisição de cadeiras de rodas para o acesso interno de pacientes;

- Eliminação de barreiras arquitetônicas.

Considerando a complexidade e importância da acessibilidade dos deficientes, principalmente em um estabelecimento público, da área da saúde, voltado ao ensino, pesquisa e assistência à comunidade, este trabalho focou esta questão tendo como palco o Campus da USP de Bauru.

\section{0 bjetivos}

Os objetivos deste estudo foram identificar, descrever e mapear barreiras físicas no Campus da Universidade de São Paulo de Bauru e apresentar as intervenções realizadas, durante o período de outubro de 2001 a dezembro de 2005. 


\section{MÉTOdO:}

\subsection{Descrição do estabelecimento, objeto do estudo de CAso}

O Campus da USP de Bauru, palco deste estudo, está localizado em uma área de $156.850 \mathrm{~m}^{2}$ e possui, aproximadamente, $45.500 \mathrm{~m}^{2}$ de área construída. É composto por três unidades:

- Hospital de Reabilitação de Anomalias Craniofaciais (HRAC): voltado à reabilitação de portadores de fissuras labiopalatais e malformações craniofaciais. Oferece, ainda, tratamento na área deaudição e visão subnormal .

- Faculdade de Odontologia de Bauru (FOB): que tem por finalidade formar profissionais nas áreas da Odontologia e Fonoaudiologia, desenvolvendo atividades de ensino, pesquisa e extensão de serviços à comunidade.

- Prefeitura do Campus Administrativo de Bauru (PCAB): desenvolve obras, reformas e manutenção dos edifícios, redes de infra-estrutura eáreas comuns (construídas e/ ou ajardinadas); segurança, transporte, limpeza pública; administração do serviço de saúde; fornecimento de refeições subsidiadas; administração do conjunto residencial estudantil; atividades esportivas, culturais, recreativas; de comunicação interna e jornalismo.

\subsection{IAGNÓSTICO DAS CONDIÇÕES EXISTENTES: IDENTIFICAÇÃO E MAPEAMENTO DAS BARREIRAS FÍSICAS E INTERVENÇÕES EXECUTADAS}

Trata-se de um estudo descritivo quantitativo, centrado em fatos objetivamente detectáveis e observáveis, para o mapeamento das condições arquitetônicas de acesso (LEOPARDI et al., 2001).

Dois bolsistas, al unos degraduação em Odontol ogia eFonoaudiologia, foram contratados para atuarem junto à CIA RP e Programa USP Legal, passando por um treinamento, ministrado por arquiteto especializado em acessibilidade, que constou da explicitação de conteúdos referentes a deficiências, implicações sociais e identificação de barreiras arquitetônicas.

Estes bolsistas, após treinamentos e com orientações fornecidas pelo representante do Programa USP Legal em Bauru, munidos do mapa do campus disponi bilizado pela Divisão de Infra-estrutura e Obras da PCAB, percorreram as áreas internas e acessos externos ao campus pontuando numericamenteas barreiras físicas e intervenções já executadas: degraus, escadas, rampas, banheiros, calçamentos comprometidos, corrimãos, etc. Com o mapa pontuado, priorizou-se as intervenções considerando as condições de fluxo de pedestres e o orçamento financeiro anual destinado ao cumprimento das adequações externas para acessibilidade dos usuários. 


\section{Resultado e discussão}

\subsection{IDENTIFICAÇÃo E MAPEAMENTO DAS BARREIRAS FíSICAS EXTERNAS}

O mapa do Campus, pontuado pelos bolsistas, identificou:

- 72 pontos de guias não rebaixadas, seguindo posições de rebaixamentos de calçada indicados pela N BR 9050 (2004): Os rebaixamentos de calçada foram localizados nas esquinas, nos meios de quadras e nos canteiros divisores de pistas.

- 21 pontos de acessos com diferenças de níveis sem rampas;

- 220 m² $^{2}$ le escadas/ rampas sem corrimãos;

- 658 m² $^{2}$ de escadas/ rampas com corrimãos em discordância com as normas vigentes;

- 03 rampas com incl inações superiores às determi nadas em normas técnicas;

- 10 banheiros parcialmente adaptados para deficientes;

- 02 vagas de estacionamento parcialmente adaptadas para deficientes;

- 02 elevadores existentes.

Asintervenções existentes foram consideradas parcial menteadaptadas porquenão atendiam, na íntegra, as normas e resoluções vigentes. N estas condições, corrimãos não atendiam al turas e diâmetros das barras determinados por normas; não possuíam barras paralelas; não possuíam cores contrastantes. As rampas existentes possuíam incl inação superior à determinação das normas. Os banheiros não possuíam espaço para a circulação de cadeira de rodas; não obedeciam as alturas de papeleira, porta papel toal ha, saboneteira, incl inação eal tura de espelho e protetor de porta. As vagas para deficientes não possuíam sinalização vertical e não atendiam às dimensões determinadas em norma.

\subsection{Intervenções Realizadas no Período de OUtubro de 2001 a dezembro de 2005}

Ao longo dos anos, anteriormente ao período estudado, algumas intervenções foram executadas, como, por exemplo, instalação de corrimãos em escadas e adaptação de banheiros. Porém, foi observado, que essas intervenções, em sua total idade, não atendiam, na íntegra, às determinações das normas vigentes, conforme discutido no tópico anterior.

A partir de 2001, de forma criteriosa, foi estabelecida uma relação de prioridades de áreas, considerando valores financeiros disponibilizados anual mente, áreas de maior fluxo de pedestres edemaiores atrações para a execução das intervenções externas: rebaixamento de guias, rampas de acesso, instalação de 
corrimão eguarda-corpo em escadas erampas, vagas para estacionamento ecentrais de atendimento ao surdo e aquisição de cadeiras de rodas.

Rebaixamento de guias ou rebaixamento de calçadas:

O Campus possui uma rua central, asfaltada, e, ao longo de seu calçamento foram executados, em 19 pontos, rebaixamentos de guias, somando $115 \mathrm{~m}^{2}$, delimitando um trajeto possível de ser transposto por cadeirante e/ ou deficiente visual. Os rebaixamentos de guias foram executados com linhas de piso tátil, pintados com tinta especial em cores contrastantes com a cor da rampa, conforme mostrado na Figura 1. Os pisos táteis instalados nas rampas foram confeccionad os pela Prefeitura da Cidade Universitária da USP. Funcionam como sinalização de alerta, possuem dimensão de $30 \mathrm{~cm} \times 35 \mathrm{~cm}$, em consonância com a NBR 9050 (2004), atendendo as especificações referentes às dimensões elocal izações. Os pisos táteis foram instalados, na faixa inferior, a 0,40m da face externa da guia, e na faixa superior, no plano, imediatamente após o final da rampa. A execução das intervenções foi real izada pelos funcionários da Divisão de Infra-estrutura da PCAB. Estas modificações asseguraram circulação livre de obstáculos efacilitaram a identificação de transição de superfície, conforme previsto nas normas técnicas (ABNT, 2004)

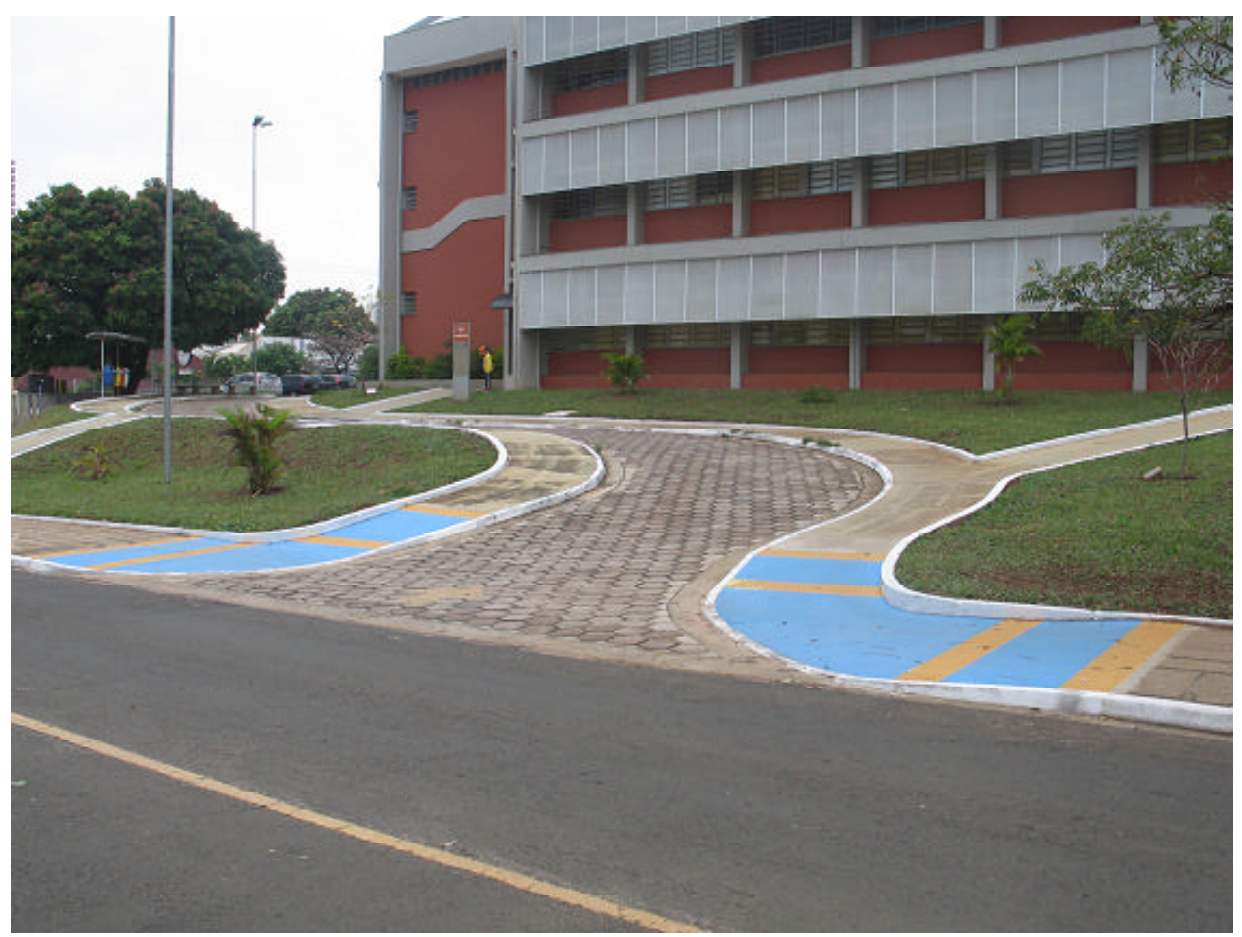

Figura 1 - Guias rebaixadas. 
Rampas de acesso:

Foram executadas rampas em 8 pontos: acesso aos prédios da Reprografia, Restaurante (em 2 pontos), Setor deManutenção, PA B Banespa, PAB N ossa Caixa, Triagem, Urgência Odontológica e acesso ao banheiro do A nfiteatro da Odontopediatria.

As rampas foram executadas (Figura 2) seguindo as especificações do Informativo Técnico USP Legal n. 12 (2002), em concordância a N BR 9050 (2004):

- Largura mínima de 1,20m; com corrimão;

- Patamares no início efinal decada segmento derampa, com no mínimo 1,20m de comprimento no sentido do movimento;

- Piso tátil para sinalização, com largura de 0,30m, instalado a 0,28m antes do início e a 0,28m após o término de cada segmento de rampa;

- Inclinação igual ou inferior a 8,33\%.

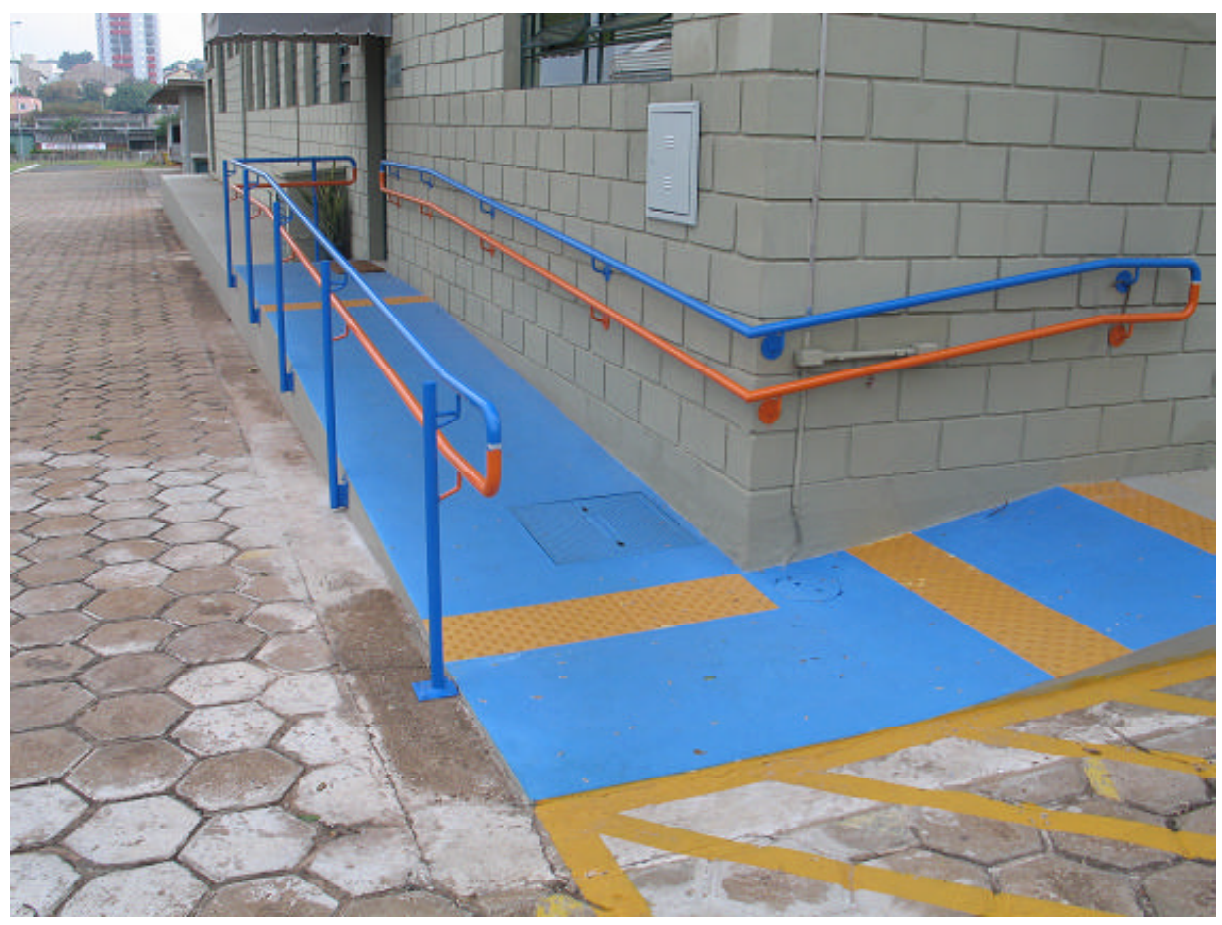

Figura 2 - Rampas e guias rebaixadas 
Equipamento de segurança: Corrimão e Guarda-corpo:

Em 14 pontos deescadas e rampas externas foram instalados corrimãos e guarda-corpos para acessos aos prédios do Restaurante, Ginásio de Esportes, Prefeitura do Campus, Registro de Diplomas, PAB BANESPA, perfazendo $137 \mathrm{~m}$ de extensão. Os corrimãos foram confeccionados atendendo o Informativo Técnico USP Legal n. 4 (2002), em consonância com a NBR 9050 (2004):

- Contínuo; em duas alturas, 0,70 m e 0,92 m e instal ado em ambos os lados;

- Com formato que permite boa empunhadura e desl izamento da mão

- Secção circular - diâmetro da barra de $3 \mathrm{~cm}$ a 4,5cm;

- Firmemente fixados e em material rígido - barras metálicas;

- Ultrapassando 0,30m antes do início e após o término das escadas ou rampas;

- Distância entre parede e face externa da barra igual a 4cm;

- Pintura em cores contrastantes - azul e laranja.

Os guarda-corpos foram confeccionados atendendo a N BR 9050 (2004), Instrução Técnica n. 11 (2004) do Decreto n. 46.076/ 01:

- Altura igual a 1,05m;

- Grade telada com malha igual a 0,10m;

- Proteção nas extremidades das Iongarinas para evitar acidentes.

Vagas para estacionamento:

O Campus da USP de Bauru possui 521 vagas de estacionamento distribuídas em diferentes áreas de bolsões. Destas vagas, 7 foram reservadas para uso exclusivo do deficiente físico, localizadas em locais estratégicos visando facilitar o acesso a prédios de maior atração. O número de vagas exclusivas é superior à determinação da NBR 9050 (2004), que estabel ece o valor de 1\% do total de vagas existentes para situações acima de 100 vagas, demonstrando, assim, a preocupação do Campus no atendimento a essa parcela da população.

Das 7 vagas, 5 foram criadas após a implantação do Programa USP Legal. Os locais priorizados foram os estacionamentos defronte aos prédios da A natomia/ Odontopediatria/ Ortodontia da FOB (2 vagas), Clínicas Odontológicas da Graduação da FOB, Urgência Odontológica, Clínica de Odontopediatria e Registro de Diplomas.

A demarcação de 5 vagas foi realizada de acordo com as orientações do Informativo Técnico USP Legal no. 2, atendendo, ainda, a NBR 9050 (2004) 
- 03 vagas perpendiculares ao meio fio e 02 vagas inclinadas a $45^{\circ}$

- Com dimensões: 5,50m x 2,50m;

- Área para transferência de 1,20m delargura, zebrada na cor amarelo com faixas de largura igual 0,10m, espaçadas a cada 0,30m;

- Sinalização horizontal de piso com o símbolo internacional de acesso, com fundo azul e pictograma branco, nas dimensões de 0,80m x 0,80m, posicionado a 0,50m do início da vaga.

Central de atendimento telefônico ao surdo:

Com o atendimento, no Campus, ao deficiente auditivo por meio da Clínica de Fonoaudiologia da FOB, Centro de Pesquisas A udiológicas, e Hospital de Reabilitação de A nomalias Craniofaciais evisando a inclusão, foram instal adas duas centrais telefônicas de atendimento ao surdo: uma, na Secretaria da Clínica de Fonoaudiologia da FOB e, outra, na Central de Agendamento do HRAC. A tecnologia utilizada beneficia a pessoa surda permitindo que, a partir deum tel efone para surdos, esta possa se comunicar. As Centrais de A tendimento ao Surdo são constituídas por equipamento acoplado em microcomputador, por um hardware e um software. Dois funcionários do Campus receberam treinamento para operar e informar sobre o equipamento e sua utilização.

Aquisição de cadeiras de rodas:

Foram adquiridas três cadeira de rodas, além das duas existentes, para que os usuários que freqüentem os espaços do campus da USP/ Bauru, possam transitar e se beneficiar dos serviços oferecidos a população.

\section{CONSIDERAÇõES FINAIS}

Este estudo mostrou a identificação e mapeamento das barreiras físicas eapresentou as intervenções queforam possíveis com os recursos anuais destinados para este fim, durante o período de outubro de 2001 a dezembro de 2005.

Vários estudos apontam a necessidade redução das barreiras arquitetônicas para promover a integração de pessoas com deficiência em todos os ambientes. Estes demonstram quena presença de barreiras a qual idade dos serviços prestados está comprometida e a legislação brasileira está desrespeitada, sendo necessário então, reconhecer os direitos legítimos e legais da acessibilidade e integração social das pessoas e promover mudanças (EMMEL; CASTRO, 2003; MANZINI et al., 2003; RODRIGUES, 2004; BITTEN COURT et al., 2004; ANDRADE et al., 2007; PA GLIUCA et al., 2007).

O trabalho desta comissão aqui apresentado é apenas uma pequena amostra da necessidadedemudanças e do compromisso assumido por estecampus para promover o intercâmbio e a acessibilidade, garantindo o direito de todos ao acesso nesta instituição pública. 
Segundo Rodrigues (2004) as barreiras arquitetônicas têm que ser vistas não somente como um conjunto de rampas e medidas a serem respeitadas, mas como uma filosofia geral de acolhimento, conforto efacilidades em todas as dependências dos edifícios.

A CIARP tem sensibilizado a comunidade uspiana local permitindo reflexões sobre valores de extrema relevância como liberdade de ir e vir, respeito à diferença e acol himento ao usuário. Promover mudanças arquitetônicas também possibilita que mais pessoas com necessidades especiais possam conviver no ambiente, o que promove a interação, mas não garante, necessariamente, a integração das pessoas. Estetrabal ho terá continuidade, para quetodos os espaços do campus da USP de Bauru sejam cada vez mais adequados para receber todas as pessoas, inclusive aquelas que apresentam necessidades especiais. Além das barreiras arquitetônicas énecessário quehaja mudanças deatitudes proporcionando novas abordagens no atendimento dos cidadãos, buscando o respeito pelas pessoas e melhoria na qualidade de vida para todos.

\section{ReferênCIAS}

ABNT NBR9050: A cessibilidade a edificações, mobiliário, espaços eequipamentos urbanos. A ssociação Brasilei ras de N ormas Técnicas, Rio de Janeiro; A BNT, 2004.

ANDRADE, M.S.A.; et al. Pessoas com deficiência rumo ao processo deinclusão na educação superior. 2007. Artigo eletrônico. Disponível em: <http:/ / www.fasb.edu.br/ revista/ index/ php/ conquer.article/ view/ pdfinterstitial 27/ 0>. A cesso em: 3 abr. 2008.

BITTEN COURT, L. S. et al.. A cessibilidadee Cidadania: barreiras arquitetônicas eexclusão social dos portadores de deficiência física. ANAISDO 20 CON GRESSO BRASILEIRO DE EXTENSÃ O UNIVERSITÁRIA, 2, Belo Horizonte, 2004. A nais... Belo Horizonte, 2004. Disponível em: বhttp:/ / www.letras.ufmg.br/ educonl/ arquivos/ anais/ congresso >. A cesso em: 3 abr. 2008.

BRASIL. Os direitos das pessoas portadoras de deficiência - Lei no 7853/ 89. Decreto $n$. 914/ 93. Brasília, DF: Corde, 1994.

BRASIL. PROJETO DE LEI (4767/ 98). N ormas gerais eCritérios básicos para a promoção da acessibilidade das pessoas portadoras de deficiência ou mobilidade reduzida. Brasília, DF, 1998.

EM MEL, E.M. G; CASTRO, C.B. Barreiras arquitetônicas no campus universitário: o caso da UFSCAR. In: MARQUEZINI, M. C. et al.. (Org.). Educação física, atividades lúdicas e acessibilidade de pessoas com necessidades especiais. Londrina: Uel, 2003. p.177-183. (Coleção Perspectivas Multidisciplinares em Educação Especial. v.9)

INFORMATIVO TÉCNICO: Vaga de auto paralela ao meio fio. USP Legal: para pessoas com deficiência. São Paulo: USP, 2002./ Informativo Técnico n. 4/ 9.

LEOPA RDI, M. T. et al. M etodologia na pesquisa na saúde. Santa M aria: Paloti, 2001. 
MANZINI, E. J. et al.. A cessibilidade em ambiente Universitário: identificação e quantificação de barreiras arquitetônicas. In: MARQUEZINI, M. C. et al. (Org.). E ducação física, atividades lúdicas e acessibilidade de pessoas com necessidades especiais. Londrina: Uel, 2003. p.185-192 (Coleção Perspectivas M ultidisci plinares em Educação Especial, v. 9).

PAGLIUCA L. M. F. et al. A cessibilidade e deficiência física: Identificação de barreiras arquitetônicas em áreas internas de hospitais de Sobral, Ceará. R ev. Esc. Enferm. v. 41, n.4, p.581-588, 2007.

RODRIGUES, D. A. Inclusão na Universi dade: Limites e possi bilidades da construção de uma universidade inclusiva. Revista de E du cação Especial da U FSM , n. 23, 2004. Disponível em: বhttp:/ / www.ufsm.br/ ce/ revistaceesp/ 2004/ al/ al.htm>A cesso em: 3abr. 2008.

SÃ O PA ULO. Secretaria de Estado dos negócios da Segurança Pública - Instrução Técnica n. 12 / 2004 - Decreto Estadual 46.076/ 01. São Paulo, 2004. 